\title{
CLINICAL AND NEUROPSYCHOLOGICAL PROFILE IN A SAMPLE OF CHILDREN WITH ATTENTION DEFICIT HYPERACTIVITY DISORDERS
}

\author{
Sueli Rizzutti', Elaine Girão Sinnes², Luzia Flavia Scaramuzza ${ }^{3}$, Lívia Freitas ${ }^{3}$, \\ Denise Pinheiro ${ }^{4}$, Sonia M. Palma $a^{5}$, Claudia Berlim Mello², Monica Carolina Miranda 2 , \\ Orlando Francisco Amadeo Bueno ${ }^{2}$, Mauro Muszkat ${ }^{7}$
}

\begin{abstract}
The aim of this study was to evaluate clinical and neuropsychological findings in children with suspicion of attention deficity hyperactivity disorder (ADHD). The assessment involved 150 children aged 7 to 14 referred to NANI at UNIFESP. Results: 75 children ( $55 \mathrm{M}$ and $20 \mathrm{~F}$ ) fulfilled the criteria for ADHD, among which 35 were of the inattentive type, 28 of combined type and 12 were hyperactive/impulsive. There was negative correlation between the digit score and the Corsi test. Children with hyperactivity and impulsivity had a low performance for functional memory. Children with oppositional defiant disorder presented pattern changes in adaptability when there was a change in the rhythm the stimuli were presented and lower adaptation to time variability (Hit RT), in addition to higher rates of omission in the continuous performance test. Conclusion: This study suggests multiple interrelations between the scores of neuropsychological battery useful for detailed delimitation of the clinical profile of children with ADHD.
\end{abstract}

KEY WORDS: attention deficit disorder, children, hyperactivity, multidisciplinary, comorbities.

\section{Perfil clínico e neuropsicológico de crianças com transtorno do deficit de atenção e hiperatividade}

Resumo - O objetivo deste trabalho foi delimitar indicadores clínicos e neuropsicológicos em crianças com suspeita de transtorno do déficit de atenção e hiperatividade (TDA/H). Foram avaliadas 150 crianças (idade de 7 a 14 anos) encaminhados ao NANI da UNIFESP. Resultados: 75 crianças ( 55 M e 20 F) preenchiam os critérios para o TDA/H, dentre os quais 35 (46,6\%) pacientes eram desatentos, 28 do tipo combinado e 12 do tipo hiperatividade/impulsivo. Observou-se correlação negativa com o escore de dígitos e no escore do teste de Corsi. Crianças com hiperatividade e impulsividade apresentaram baixo desempenho nas funções relacionadas à memória operacional. Crianças com transtorno opositor desafiante apresentaram alterações nos padrões de adaptação às mudanças do ritmo com menor adaptação às variações do tempo de exposição aos estímulos (Hit RT), além de maiores taxas de omissão no teste contínuo de performance. Conclusão: Este estudo sugere múltiplas interrelacões entre os escores em provas neuropsicológicas que são úteis para uma delimitação do perfil clínico de crianças com TDAH.

PALAVRAS-CHAVE: transtorno do déficit de atenção, crianças, hiperatividade, multidisciplinar, comorbidades.

The attention deficit hyperactivity disorder (ADHD) is one of the most frequent neurodevelopmental disorders, with an estimated occurrence of $3 \%$ to $5 \%$ in schooling children ${ }^{1,2}$. Such oscillation is partially due to the heterogeneity and complexity of the required criteria for a precise diagnosis, which involves neuropsychological aspects as well as the delimitation of organic and neurobiological bases ${ }^{3,4}$. Environmental risk factors are considered fundamental in epidemiological studies on ADHD because socio-demographic variables such as the number of siblings, educational level of the family, comorbities with neuropsychiatrical disorders, aggressive behaviour of parents as well as maternal mental disorders seem to influence the expressions of ADHD ${ }^{5,6}$. The neurobiology of ADHD has been the object of many studies - especially since the early 90's - which suggested multiple influences related to the modulation and expression of dopaminergic and noradrenergic neurotransmitters of genet-

Departamento de Psicobiologia, Universidade Federal de São Paulo, São Paulo SP, Brazil: ${ }^{C}$ Child Neurologisty; ${ }^{2}$ Neuropsychologist; ${ }^{3}$ Psychologist; ${ }^{4}$ Social Worker; ${ }^{5}$ Psychiatrist.

Received 13 June 2008, received in final form 8 August 2008. Accepted 9 September 2008.

Dra. Sueli Rizzutti - Rua Embaú 54 -04039-060 São Paulo SP - Brasil. E-mail: surizzutti@ig.com.br 
ic and neuromaturational bases. It is believed that several genes of small effect as well as environmental agents are responsible for such genetic vulnerability for this disor$\mathrm{der}^{7,8}$, as well as structural abnormalities and neurochemical dysfunction related to subcortical, parietal and frontal circuits ${ }^{9,10}$. Inattention, hyperactivity and impulsivity symptoms are considered cardinal symptoms of ADHD and compose the criteria of DSM-IV ${ }^{11}$. In order to be considered characterised, these symptoms must be accompanied by clinical expression affecting social interaction, quality of life and cognitive or affective/emotional performance of the individual.

Thus, ADHD is characterized by a range of symptoms not always clear and not easily distinguishable from other psychiatrical (in more serious cases) or normality (in milder cases) disorders ${ }^{12}$. Overall, individuals with ADHD tend to present learning difficulties, behavioural and conduct disorders, mood disorders, motor disorders and delay of speech development. Although the majority of individuals present symptoms of inattention as well as hyperactivity and impulsivity, there are individuals who show a predominance of one or a few cognitive-behavioural patterns. For many authors, the DSM-IV-TR overestimates the diagnosis, as many of the evaluated children would be excluded when broadening the scope of assessment through a detailed neuropsychological test intended to delimit several areas involved such as selective, alternating and sustained attention, in addition to common executive dysfunctions in $\mathrm{ADHD}^{13}$. Many studies have recommended a neuropsychological evaluation for diagnostic and complementary determination of ADHD cases ${ }^{5}$. Sustained attention tests such as the Continuous Performance Test $(C P T)^{14,15}$, in addition to a broad spectrum of cognitive tests, scales and questionnaires have been utilised in order to determine specific deficits with emphasis on predominant disorders in associative areas not only related to attention but also to functional memory and cognitive flexibility. However, the determination of which tools are the most appropriate and sensitive to the clinical heterogeneity of ADHD remains controversial, as well as the delimitation of the clinical and neuropsychological indicators utilised in the protocol of children with suspicion of ADHD.

The aim of this study is to analyse the clinical and neuropsychological characteristics of the children referred to the ADHD Outpatient Sector at NANI (Núcleo de Atendimento Neuropsicológico Infantil Interdisciplinar da Universidade Federal de São Paulo) in order to delimit the clinical and neuropsychological indicators utilised in the protocol of children with suspicion of ADHD.

\section{METHOD}

The Ethics Committee approved the present study and an informed consent was obtained from all parents before enrollment.
One hundred fifty children, aged 7 to 14 , were selected through voluntary enrolment by the parents at the ADHD Outpatient Sector at NANI-UNIFESP following complaints of restlessness, attention difficulties and/or impulsivity. They were then called for an individual trial interview. Patients with complaints related to pervasive developmental disorders and evident neuropsychomotor global development delay. Children who met the inclusive criteria (at least 6 out of the criteria defined by DSM-IV for hyperactivity and/or 6 for inattention starting before the age of 7) were referred to a multidisciplinary assessment schedule which consisted of medical and neuropsychological evaluation, as well as social and family assessment.

Out of the 150 children assessed in the trial interview with attention difficulties, psychomotor restlessness and adaptive difficulties related to impulsivity, negativism and aggressiveness, 124 were selected and 26 were excluded as they had complaints related to invasive disorders of development, evident neuropsychomotor global development delay and mental disability.

They were then submitted to detailed neurological and psychiatrical examination and neuropsychological evaluation.

The neuropsychological protocol included:

1) Intellectual Level Assessment: (Wechsler Children Intelligence Scale WISC-III abbreviated) ${ }^{16}$. 2) EACI-P Scale ${ }^{17}$. Scale answered by the teacher, who assesses five main areas of child behaviour: Hyperactivity/Conduct Problems (EACP-I), Independent Functioning (EACIP-II), Inattention (EACIP-III), Neuroticism/Anxiety (EACIP-IV) and Social Interaction (EACIP-V). 4) Computerized Attention Test: CPT - Conners' Continuous Performance Test ${ }^{14}$ - assesses sustained attention abilities and mental flexibility. The Conners' CPT is a computerized visual task that requires distinguishing non-target (X letters) and target (non-X letters) stimuli. The child is instructed to press the computer keyboard for any key that appears on the screen, except for letter $X$. The programme generates several measures indicating inattention, impulsivity or sustained attention problems. All measures are converted into $t$-scores and percentiles ${ }^{18}$. 5) Working memory ${ }^{19}$ assessed through: a) forward and backward Digit Span, assesses the phonological loop component of working memory; b) forward and backward Corsi blocks: assesses the ability to reproduce a sequence of stimuli (blocks) in a visuo-spatial design assessing the sketchpad component of working memory. 6) Rey Figure: it is a task that assesses the visual constructive functions (Rey Copy) as well as visual memory (Rey Memory) through a guided reproduction from memory of a complex figure ${ }^{20}$.

Children presenting symptoms of inattentive, hyperactiveimpulsive and combined ADHD types, with or without comorbities, were included in the study.

\section{Statistical analysis}

The results of clinical and neuropsychological tests were compared through analysis of variance (ANOVA), followed by the Tukey test and Pearson's correlation coefficient for the assessment of correlations between clinical and neuropsychologi- 
Table 1. Clinical diagnosis and gender distribution.

\begin{tabular}{lccc}
\hline Clinical characteristics & $\mathrm{n}(\%)$ & \multicolumn{2}{c}{ Gender } \\
\cline { 3 - 4 } & & $\mathrm{M}$ & $\mathrm{F}$ \\
\hline ADHD & $75(60.4 \%)$ & 55 & 20 \\
ADD - Inattentive subtype & $35(46.6 \%)$ & 17 & 18 \\
ADHD - Combined subtype & $28(37.3 \%)$ & 27 & 1 \\
Hyperactivity/impulsivity & $12(16 \%)$ & 11 & 1 \\
predominance & & & \\
No ADHD & $49(39.5 \%)$ & 35 & 14 \\
Learning disabilities & $7(14.3 \%)$ & 4 & 3 \\
Alterations in family dynamics & $10(20.4 \%)$ & 6 & 4 \\
Mental disability (‘85) & $16(32.5 \%)$ & 9 & 7 \\
Adaptive difficulties & $16(32.6 \%)$ & 10 & 6 \\
ADHD with comorbities & $33(44 \%)$ & 20 & 13 \\
Anxiety disorder & $22(66.6 \%)$ & 15 & 7 \\
Oppositional defiant disorder & $8(24.2 \%)$ & 6 & 2 \\
Conduct disorder & $3(9 \%)$ & 3 & 0 \\
Bipolar disorder & $3(9 \%)$ & 2 & 1 \\
Depression & $3(9 \%)$ & 2 & 1 \\
\hline
\end{tabular}

M, male; F, female.

cal variables. Significant differences were taken into account assuming a significance level of $5 \%$.

\section{RESULTS}

Most children were males (104: 80.6\%), with an average age of 7 years and 7 months, ranging from 6 to 12 (sd 1.6). With regards to the DSM IV criteria for ADHD, 75 children $(60.4 \%)$ met at least 6 criteria for inattention and/or hyperactivity, whereas 35 of them (46.6\%) were predominantly inattentive; $37,3 \%$ (28 cases) had combined symptoms of hyperactivity and inattention and 12 (16\%) had hyperactivity plus impulsivity. From the remainder of the sample of 124 children, 49 (39.5\%) did not meet the criteria for ADHD, out of which 7 presented learning disorders (14.2\%), 10 (20.4\%) had alteration in family dynamics, 16 had adaptive disorders (32.6\%), and $16(32.6 \%)$ had mental deficit $(\mathrm{Q}<85)$ (Table 1).

Out of the 75 children who met the criteria for inattentiveness and/or hyperactivity according to DSM-IV, 33 cases (44\%) had complaints of comorbities, whereas 22 cases $(66.6 \%)$ presented anxiety disorder, 8 patients (24.2\%) had oppositional defiant disorder and 3 cases had conduct disorder (9\%). With regards to mood disorders, it was observed bipolar disorder in 3 cases (9\%) and 3 cases met the criteria for severe depression (9\%). The remaining comorbities (11 cases - 33.3\%) were linked to dysfunctional behaviour related to disturbed family dynamics, alcoholism, and exposure to domestic violence. Difficulties at school not associated to a learning disabilities were observed in 15 patients (45.4\%) (Table 1).

Table 2 shows the mean scores for the neuropsychological tests in the three groups. The EACIP Scale scores showed that there were differences for hyperactivity when the inattentive and combined groups were compared with children who did not meet the criteria for ADHD, a higher score in the combined group was then found. Inattentiveness and social interaction did not reach statistical significance (Table 2).

In relation to neuropsychological tests, it was observed that the mean score for the Rey Complex Figure copy was significantly lower in the combined group when compared to the inattentive only group and a higher score for the group without ADHD. Both groups, combined and inattentive, were significantly inferior compared to the group without ADHD, with a lower performance from the combined group.

With regards to the global cognitive performance, an average IQ of 100 was found (ranging from 80 to 126).

With regards to visual-constructive ability and visualspatial memory assessed by the Rey Complex Figure, both groups had a lower performance when compared to the group without ADHD, with a significantly lower performance from the inattentive group (40.6 SD=8) (Table 2).

The performance in the CPT showed difficulties in measures such as omission, commission, higher reaction time, higher variability scores and higher perseverance in children with ADHD when compared to children without ADHD. However, the number of omissions was significantly higher for children from the inattentive group, when compared with the ones from the combined group, which suggests that the omission measure may be a differential criterion.

Table 3 shows the correlation analysis between the clinical symptoms of inattentiveness (more than 6 criteria from DSM-IV), hyperactivity (more than 6 criteria from DSM-IV), impulsivity (more than 2 criteria from DSM-IV), with oppositional and conduct disorder in relation to the performance at CPT. Only children with oppositional and conduct disorder presented significant correlation for omission, Hit RT (SE), Hit RT Block change and variability score. Children with impulsivity presented lower variability in response style (-0.54). No difference was found for the other CPT measures in the group with predominantly inattentive or hyperactive children.

Table 4 shows the correlation analysis between the CPT measures and the neuropsychological tests for children with ADHD (combined and inattentive). Negative correlation between forward and backward digit span (phonological working memory) with omission scores $(-0.65$, -0.63 ), negative correlation between reaction time (Hit 
Table 2. Distribution of mean scores of neuropsychological tests.

\begin{tabular}{|c|c|c|c|c|}
\hline Neuropsychological assessment & $\begin{array}{c}\text { No ADD }(n=49) \text { Group } 1 \\
(\text { Mean+SD) }\end{array}$ & $\begin{array}{l}\text { ADHD }(n=40) \text { Group } 2 \\
\text { (Combined) (Mean+SD) }\end{array}$ & $\begin{array}{c}\text { ADD (n=35) Group } 3 \\
\text { (Inattentive) (Mean+SD) }\end{array}$ & $\begin{array}{l}\text { ANOVA } \\
\text { (Tukey) }\end{array}$ \\
\hline EACIP - Hyperactivity & $60.8(22)$ & $82.8(28.3)$ & $57.9(45.8)$ & $* * * * * * *$ \\
\hline EACIP - Inattentiveness & $14.6(85)$ & $15.6(7.6)$ & $15.0(8.6)$ & NS \\
\hline EACIP - Negative social interaction & $9.2(5.4)$ & $8.2(5.4)$ & $4.3(4.5)$ & NS \\
\hline Rey figure copy & $60(11)$ & $32.3(12)$ & $44.6(11)$ & $* . * * . * * *$ \\
\hline Rey figure memory & $80.8(13)$ & $51.8(13)$ & $40.6(8)$ & $* . * * . * * *$ \\
\hline Digits (backward) & $4(1.3)$ & $2.6(1.7)$ & $2.1(1.0)$ & NS \\
\hline Digits (forward) & $5(0.6)$ & $4(0.6)$ & $3.8(0.7)$ & NS \\
\hline Corsi (forward) & $4.3(0.6)$ & $4.4(0.7)$ & $4.1(0.6)$ & NS \\
\hline Corsi (backward) & $4.0(1.1)$ & $3.0(1.2)$ & $2.3(1.2)$ & NS \\
\hline CPT omissions & $12.2(12.7)$ & $30.2(28.7)$ & $52.9(34.2)$ & $* . * * . * *$ \\
\hline CPT commissions & $12.3(8)$ & $21.5(8)$ & $25.3(5.2)$ & $* * *$ \\
\hline Reaction time & $337.3(80)$ & $537.3(100)$ & $641.1(90.3)$ & $* * *$ \\
\hline Variability & $21.5(20)$ & $41.5(22)$ & $58.1(13.3)$ & $* . * *$ \\
\hline Perseverations & $11.1(12)$ & $20.1(12)$ & $19.3(12.6)$ & $* . * *$ \\
\hline
\end{tabular}

*Significant difference between Groups 1 and 2; **Significant difference between Groups land 3; ***Significant difference between Groups 2 and 3 ; NS non significant.

Table 3. Pearson's correlations between clinical symptoms and CPT measures in the combined and inattentive group.

\begin{tabular}{lcccc}
\hline & Inattention & Hyperactivity & Impulsivity & Oppositional/conduct \\
\hline Omissions & -0.12 & 0.06 & 0.04 & $0.45^{*}$ \\
Commissions & -0.06 & -0.08 & -0.05 & 0.31 \\
Hit RT & -0.01 & -0.01 & -0.24 & 0.09 \\
Hit RT std error & -0.10 & 0.05 & 0.04 & $0.40^{*}$ \\
Hit RT block change & 0.09 & 0.30 & 0.31 & $0.38^{*}$ \\
Hit RT block change (SE) & 0.18 & 0.33 & 0.11 & 0.31 \\
Hit RT ISI change & -0.03 & 0.24 & 0.26 & 0.23 \\
Hit RT ISI change (SE) & -0.08 & 0.06 & -0.02 & 0.34 \\
Variability & -0.15 & 0.07 & 0.12 & $0.41^{*}$ \\
Detectability & 0.23 & 0.15 & 0.12 & -0.17 \\
Perseverations & 0.16 & 0.01 & 0.09 & 0.33 \\
Response style & -0.32 & -0.01 & $-0.54^{*}$ & -0.07 \\
\hline
\end{tabular}

HT RT, Hit reaction time; Hit RT std error, Hit reaction time standard error; Hit RT block change, Hit reaction time block change; HIT block change SE, Hit standard error block change; HIT RT ISI change, Hit reaction time ISI change; Hit ISI change (SE), Hit standard error ISI change; * $\mathrm{p}<0.05$.

RT, Hit RT SE) scores and forward digit span (-0.64) and backward visuo-spatial working memory (Corsi test) were found $(-0.64)$. Thus, children with high scores for hyperactivity and impulsivity in behavioural scales had low performance in working memory tasks. The Rey Complex Figure memory reproduction was negatively correlated with the score for perseveration in the CPT $(-0.38)$.

\section{DISCUSSION}

Although the clinical diagnosis for ADHD is based on symptoms which are systematised by international scales such as DSM-IV, the multidisciplinary assessment proce- dure allows us to make diagnosis based on a more precise neurobiological basis, essential for differential diagnosis for other development disorders.

Prevalence studies have found, in clinical samples, a rate of 2 boys to 1 girl, and up to 9:1 $1^{2,3}$, also showed that prevalence is high for boys - approximately $9.2 \%$ - whereas for girls the rate is $3 \%$. In our study we found a male predominance of 3:1.

The association of ADHD with several comorbities such as oppositional defiant disorder, anxiety disorder, depression and learning disabilities also depend on a more 
Table 4. Pearson's correlations between CPT measures and IQ and neuropsychological measures in the combined and inattentive group.

\begin{tabular}{|c|c|c|c|c|c|c|c|}
\hline & $\operatorname{Rey}(\mathrm{C})$ & Rey (M) & Corsi (Fwd) & Corsi (Bwd) & Digits (Fwd) & Digits (Bwd) & Mean IQ \\
\hline Omissions & -0.11 & -0.10 & -0.19 & -0.41 & $-0.65^{*}$ & $-0.63^{*}$ & -0.18 \\
\hline Commissions & -0.09 & -0.20 & -0.01 & -0.12 & 0.16 & 0.06 & -0.33 \\
\hline Hit RT & -0.12 & 0.01 & -0.48 & $-0.64^{\star}$ & $-0.64^{*}$ & -0.33 & 0.03 \\
\hline Hit RT (SE) & -0.02 & -0.06 & -0.33 & -0.48 & $-0.67^{*}$ & -0.53 & -0.09 \\
\hline Hit RT block change & 0.01 & 0.07 & -0.00 & 0.40 & 0.39 & -0.09 & -0.21 \\
\hline Hit RT block change (SE) & 0.07 & -0.01 & 0.05 & 0.43 & 0.10 & -0.09 & 0.08 \\
\hline Hit RT ISI change & -0.00 & -0.03 & -0.28 & -0.54 & -0.48 & -039 & -004 \\
\hline Hit RT ISI change (SE) & -0.02 & -0.11 & -0.19 & -0.47 & -0.33 & -0.25 & -0.02 \\
\hline Variability & -0.31 & -0.04 & -0.19 & -0.45 & -052 & -0.54 & -0.07 \\
\hline Detectability & -0.02 & 0.01 & -0.07 & -0.04 & -0.26 & -0.23 & 0.15 \\
\hline Perseverations & -0.31 & $-0.38^{*}$ & -0.13 & -0.27 & -0.46 & -0.40 & -0.20 \\
\hline Response style & 0.20 & 0.15 & -0.41 & -0.01 & -0.38 & -0.17 & 0.14 \\
\hline
\end{tabular}

HT RT, Hit reaction time; Hit RT std error, Hit reaction time standard error; Hit RT block change, Hit reaction time block change; HIT block change SE, Hit standard error block change; HIT RT ISI change, Hit reaction time ISI change; Hit ISI change (SE), Hit standard error ISI change; * $<0.05$.

comprehensive multidisciplinary assessment. In our study we found that, although the children met the symptomologic ADHD criteria, after completing the assessment with a detailed protocol in $39.5 \%$ of the cases (Table 1) they did not meet broader neuropsychological and clinical criteria. Such finding emphasises the risk of basing ADHD diagnosis on behavioural scales which are not totally precise for the detection of the 3 subtypes, even the behavioural scales answered by the teachers. In this study, the hyperactivity variable measured by the EACIP scale was the only one to have a positive correlation with the final diagnosis of ADHD (Table 2).

Several studies show a high prevalence of comorbities, around 20 to $50 \%{ }^{1,21}$. In our study, the presence of comorbities was found in $44 \%$ of the diagnosed cases, which shows the importance of an interdisciplinary approach as there are many disorders associated to ADHD, such as anxiety and conduct disorders, which require different clinical interventions and differentiated pharmacological treatment. We also found that comorbity cases with conduct and oppositional disorders were the ones that presented the highest number in the scores related to a lower performance on the CPT (Table 3). This reinforces the idea that comorbities have a higher impact on the cognitive and attention performance of an individual with ADHD.

In recent years, the role of multidisciplinary evaluation on ADHD has been constantly reviewed and has drawn the attention of several centres from different countries ${ }^{5,6,12}$. Hence, the issue is currently the reach of news directions for more precise diagnostic procedures. The multidisciplinary approach aims to delimit better the level of interference of ADHD symptoms on social, affective, edu- cational functioning, and to identify the more relevant professional, family and educational interventions in each case $^{6,12}$. For this purpose, it is essential to gather information on family dynamics, socio-economic and the educational conditions of patients. These information may provide evidence on the impact of both the disorder itself and the treatment have on development and quality of life of children.

The rising of various hypotheses linked to the etiology and the neuropsychological impact on ADHD has generated several studies and efforts towards the determination of the clinical advantages of neuropsychological tests in multiple areas related to motor inhibition ${ }^{14}$, planning and organization (Rey Complex Figure ${ }^{20}$ ) and working memory (Digit repetition and Corsi blocks ${ }^{19}$ ). The lack of a protocol that will determine a gold standard in clinical and neuropsychological assessment of ADHD reinforces the need for studies that establish objective multiple variables, helping define which tools are the most appropriate in the diagnosis of children with ADHD.

One of the most utilized tests in the assessment of sustained attention and inhibition is the Conners' CPT Test, not only because it is easy to manage but also for its broad profile allowing age group distribution for the performance analysis parameters, such as reaction time, omission and commission errors closely related to attention ability.

In our study, the Conners' CPT Test revealed to be sensitive enough for delimiting cases that meet criteria for a clinical ADHD profile against non-ADHD profiles, but not for an absolute differentiation between the ADHD subtypes, since the it was found significant differences only 
for omission scores (Table 2). Children with ADHD presented a slower reaction time, more omission and commission errors when compared to children without ADHD, similarly to the findings in literature ${ }^{22,23}$. The specificity of the existing variables in the CPT is quite contradictory and sometimes inconsistent, especially when differentiating the ADHD subtypes ${ }^{24,25}$. We also must consider that small differences found in the correlations between the ADHD subtypes may have been influenced by the relatively small number cases in the hyperactive/impulsive subtype sample, but such finding is in line with those of other authors ${ }^{5,26}$, who report that although the CPT has high sensitivity (about $88 \%$ when detecting ADHD), it has low specificity (from 20 to 37\%) when identifying the different subtypes. On the other hand, in our study, the cases of ADHD which meet the criteria for oppositional and/or conduct disorder presented a higher frequency for omission, higher reaction time, Hit RT Block change and variability (Table 3).

The Conners'CPT Test as an isolated paradigm is not ideal for the diagnosis of subtypes, but it is useful in the assessment on the overall impact that ADHD has on cognitive domain, in the separation of purely contextual cases and is also particularly sensitive in cases of comorbity such as oppositional defiant and conduct disorders (Table 3).

The negative correlations (Table 4) observed in our study between the functional memory scores (Digits Span and Corsi Blocks tests) and omissions and commissions in the Conners' CPT reinforce the important role of functional memory as the origin of cognitive and behavioural functions associated to ADHD ${ }^{12,27}$.

As for the visual-constructive and visual-spatial abilities assessed through the Rey Complex Figure, we found negative correlation between the percentile of perseveration in the Conners'CPT and the reproduction from memory of the figure shown. The higher the performance on the memory test, the lower was the perseveration in the CPT (Table 4). We considered that such result is related to the executive function in children with ADHD. Homack and Riccio ${ }^{28}$ and Shin and $\mathrm{Kim}^{29}$ claim that the Rey Complex Figure is a very adequate test to delimit patterns of spatial planning/organization, but is not specific enough to differentiate the ADHD subtypes. The reliability of performance in the Rey Complex Figure, which requires spatial planning and visual-constructive abilities, proved to be an interesting test to assess attention interference of spatial functions. This way, the copy and memory modules from the Rey Figure may reflect the modulation of visual selective attention and self-monitoring, which are essential to the organization and planning of complex activities ${ }^{20}$ suggesting that performance flaws related to a higher score for perseveration in the CPT may reflect an executive dysfunction.

When separating those children with at least 6 criteria for inattentiveness, we observed a strongly negative correlation between the occurrence of inattentiveness as per the DSM IV scale and the response style, with higher mean reaction time in inattentive children (with more than 6 criteria for inattentiveness). Children with ADHD of combined type, when compared to children of inattentive type, had a lower performance on the Conners' CPT by higher omission errors (Table 2). With regards to impulsivity, we also observed that the more impulsive they were (score of 3 DSM-IV criteria for impulsivity) the higher was the reaction time and the variability in the response style (Table 3).

In combined ADHD cases (score $>6$ for hyperactivity), performance correlated positively in higher omissions, reaction time and variability of answers in the CPT (Table 2).

Overall, our study suggests multiple interrelations between the scores of neuropsychological tests, which are useful for a more detailed delimitation of the clinical profile of children with ADHD. In addition, better defined tests, involving not only attention and motor planning, may determine different neuropsychological profiles and, consequently, different approaches in cognitive rehabilitation and even in the selection of cases subject to pharmacological treatment. The amplification of our sample through the assessment of those cases submitted to treatment with stimulants will help us determine which clinical and neuropsychological indicators will lead us to a precise nosological delimitation, not only in order to diagnose but also to select, on a neurobiological basis, which strategies in cognitive rehabilitation are more contextualized and particular and to which extent and degree the pharmacological approach is indispensable for a better social insertion, both at home and at school, of children with ADHD.

\section{REFERENCES}

1. Garland AF, Hough RL, Mccabe KM, Yeh M, Wood PA, Aarons GA Prevalence of psychiatric disorders in youths across five sectors of care. J Am Acad Child Adolesc Psychiatr 2001;40:409-418.

2. Faraone SV, Sergeant J, Gillberg C, Biederman J. The worldwide prevalence of ADHD: is it an American condition? World Psychiatry 2003;2:104-113.

3. Brown RT. Prevalence and assessment of attention-deficit/hyperactity disorder in primary care settings. Pediatric 2001;3:39-43.

4. Gaiao AA. Hiperatividade em meninas. Um estudo de prevalência diagnostica e avaliação psicológica. João Pessoa: Idéia Editora 2001:21.

5. Palumbo DR, Diehl J. Managing attentional disorders. In Hunter SJ, Donders J (eds). Pediatric neuropsychological intervention. Cambridge University Press, 2007:253-286.

6. Smucker W, Hedayat M. Evaluation and treatment of ADHD. Am Fam Physician 2001;64:817-829.

7. Thapar A, Holmes J, Poulton K, Harrington R. Genetic basis of attention-deficit and hyperactivity. Br J Psychiatry 1999;174:105-111. 
8. Castellanos FX. Neuroimaging studies of ADHD. In Solanto MV, Arnsten AFT and Castellanos FX (eds). Stimulant drugs and ADHD: basic and clinical neuroscience. New York: Oxford University Press, 2001:248.

9. Busha G, Valeraa EM, Seidmana LJ. Functional neuroimaging of attention-deficit/hyperactivity disorder: a review and suggested future directions. Biol Psychiatry 2005;57:1273-1284.

10. Seidman LJ, Valeraa EM, Makrisg N. Structural brain imaging of attention-deficit/hyperactivity disorder. Biol Psychiatry 2005;57:1263-1272.

11. American Psychiatry Association. DSM-IV. Manual diagnóstico e estatístico de transtornos mentais. 4.Ed. Porto Alegre, Artes Médicas, 1995.

12. Barkley RA. Attention deficit hyperactivity disorder: a handbook for diagnosis and treatment, 3.Ed. New York: Guilford Press, 2005.

13. Guardiola A, Fuchs FD, Rotta NT. Prevalence of attention-deficit hyperactivity disorders in students. Arq Neuropsiquiatr 2000;58:401-407.

14. Conners CK. Conner's continuous performance test. Toronto: MultiHealth System, 2000.

15. Epstein JN, Erkanli A, Conners CK. Relations between continuous performance test performance measures and ADHD behaviors. J Abnorm Child Psychol 2003;31:543-554.

16. Wechsler D. Escala de Inteligência Wechsler para crianças. Terceira Edição. Padronização Brasileira: Vera LM Figueiredo. São Paulo. Casa do Psicólogo, 2002.

17. Brito GNO. EACI-P - Escala de avaliação do comportamento infantil para o professor. Manual. São Paulo: Ed. Entreletras, 1999.

18. Miranda MC, Sinnes EG, Pompéia S, Bueno OFA. A comparative study of performance in the conner's continuous performance test between Brazilian and American children (online first) 2007, doi 10.1177/10870 54707299412

19. Santos FH, Mello CB, Bueno OFA. Cross-cultural differences for three visual memory tasks in Brazilian children. Percept Mot Skills 2005;101:421-433.
20. Meyers JE, Meyers KR. Rey comples figure test and recognition trial. Professional manual. Odessa, FL: Psychological Assessment Resource, 1995.

21. Tramontina S, Schmitz M, Polanczyk G, Rohde LA. Juvenile bipolar disorder in Brazil: clinical and treatment findings. Biol Psychiatry 2003;53:1043-1049.

22. Losier BJ, Mcgrath PJ, Klein RM. Error patterns on the continuous performance test in non-medicated and medicated samples of children with and without ADHD: a meta-analytic review. J Child Psychol Psychiatry Allied Discipl 1996;37:971-987.

23. Riccio CA, Reynolds CR. Continuous performance tests are sensitive to ADHD in adults but lack specificity: a review and critique for differential diagnosis. Ann N Y Acad Sci 2001;931:113-139.

24. Schatz AM, Ballantyne AO, Trauner DA. Sensitivity and specificity of a computerized test of attention in the diagnosis of attention-deficit/ hyperactivity disorder. Assessment 2001;8:357-365.

25. Preston AS, Fennell EB, Bussing R. Utility of a CPT in diagnosing ADHD among a representative sample of high-risk children: a cautionary study. Child Neuropsychol 2005;11:459-469.

26. Rielly NE, Cunningham CE, Richards JE, Elbard H, Mahoney WJ. Detecting attention deficit hyperactivity disorder in a communications clinic: diagnostic utility of the Gordon diagnostic system. J Clin Exp Neuropsychol 1999;21:685-700.

27. Brocki KC, Bohlin G. Developmental change in the relation between executive functions and symptoms of ADHD and Co-occurring behaviour. Inf Child Dev 2006;15:19-40.

28. Homack S, Riccio CA. A meta-analysis of the sensitivity and specificity of the Stroop Color and Word Test with children. Arch Clinic Neuropsychol 2004;19:725-743.

29. Shin MS, Kim YH, Cho SC, Kim BN. Neuropsychologic characteristics of children with attention-deficit hyperactivity disorder (ADHD): learning disorder, and tic disorder on the Rey-Osterreith Complex Figure. J Child Neurol 2003;18:835-844 